\section{Avaliação de medidas de controle da esquistossomose mansoni no Município de Sabará, Minas Gerais, Brasil, 1980-2007}

\author{
Evaluation of schistosomiasis mansoni control \\ measures in Sabará, Minas Gerais State, Brazil, \\ 1980-2007
}

Cíntia Honório Vasconcelos 1 Paula Cristina Marques Cardoso ${ }^{1}$ Wesley Caldeira Quirino ${ }^{1}$ Cristiano Lara Massara 1 Graciela Larissa Amaral 1 Ricardo Cordeiro 2 Omar dos Santos Carvalho 1

\title{
Introdução
}

${ }^{1}$ Centro de Pesquisa René Rachou, Fundação Oswaldo Cruz, Belo Horizonte, Brasil. 2 Faculdade de Ciências Médicas, Universidade Estadual de Campinas, Campinas, Brasil.

Correspondência C. H. Vasconcelos Laboratório de Helmintologia e Malacologia Médica, Centro de Pesquisa René Rachou, Fundação Oswaldo Cruz. Av. Augusto de Lima 1715 Belo Horizonte, $M G$ 30190-002, Brasil. cintiavasc@cpqrr.fiocruz.br

\begin{abstract}
Schistosomiasis control programs in Brazil have helped reduce prevalence and the severe forms of the disease, but have failed to prevent new foci from appearing, especially on the periphery of large cities. The current article aims to assess the prevalence of schistosomiasis and the presence of intermediates hosts for Schistosoma mansoni in the district of Ravena, Municipality of Sabará, Minas Gerais State, Brazil, 27 years after implementing water treatment and specific treatment for infected individuals. Ravena responded positively to schistosomiasis control measures, with a prevalence of $2.5 \%$. However, the district remains a potential risk area, since it displays favorable environmental and ecological factors for maintenance of the disease. For the prevalence rates to continue low, it will be necessary to invest in sanitation and health education, in addition to treatment of infected individuals.
\end{abstract}

Communicable Disease Control; Schistosomiasis mansoni; Mollusca
No Brasil, como em outros países, ocorreu uma importante mudança no quadro da esquistossomose em razão dos programas de controle implementados a partir da década de 1970 1,2. Esses programas têm contribuído para a redução da prevalência e das formas graves da esquistossomose, mas não têm impedido que novos focos da doença apareçam ${ }^{3,4}$.

Um dos principais motivos para a expansão da endemia é a prática do turismo rural 5 e a migração de pessoas infectadas, especialmente para as áreas periféricas dos grandes centros urbanos do país 3,6.

Fatores sócio-econômicos e sócio-demográficos também são determinantes na transmissão da doença, sendo imprescindível levá-los em consideração antes de se decidir por qualquer medida. Vários pesquisadores 7,8,9,10 observaram a associação de tais fatores com a ocorrência da esquistossomose. Estudos realizados em áreas urbanas e rurais de diversas regiões endêmicas do país encontraram as mesmas dificuldades em relacionar fatores sócio-econômicos com risco de infecção por Schistosoma mansoni por causa da homogeneidade das condições sociais e sanitárias das populações em estudo. Apesar das dificuldades encontradas, pesquisadores obtiveram como variáveis preditoras significantes a idade, $\mathrm{o}$ contato com água não tratada, o número de pessoas por residência, o tipo de moradia e a baixa 
escolaridade do chefe da casa 10 . Essas variáveis apontaram os fatores sócio-econômicos como forma de direcionar as medidas de controle da esquistossomose.

No estudo realizado na década de 1980, no distrito de Ravena, Município de Sabará, Estado de Minas Gerais, Brasil, foram identificados fatores sócio-econômicos e comportamentais da população relacionados ao risco de infecção por S. mansoni para um posterior fornecimento de água potável e tratamento quimioterápico. Foi constatada uma prevalência de $36,7 \%$; crianças até 14 anos apresentaram as maiores médias de ovos por grama de fezes. Os determinantes de risco identificados para a transmissão da doença estavam relacionados às atividades de lazer em córregos e clubes recreativos localizados nos arredores do distrito 11 . Neste mesmo ano, após a implantação do sistema de água tratada a toda população, foi dado início a tratamentos quadrienais dos infectados (1980, 1984, 1988 e 1992) 12. Foi observada, no decorrer do estudo, uma redução da prevalência para $11,5 \%$ da carga de ovos e também da morbidade, entretanto não foi possível apontar se a associação de medidas ou se elas aplicadas individualmente foram responsáveis por essa redução.

O presente artigo tem como objetivo primeiro avaliar a prevalência da esquistossomose e a presença de hospedeiros intermediários do S. mansoni no distrito de Ravena no período de 1980 a 2007 e discutir possíveis fatores que podem contribuir para sua manutenção.

\section{Métodos}

\section{Área de estudo}

Ravena é um distrito do Município de Sabará situado a $30 \mathrm{~km}$ de Belo Horizonte, pertencente à região metropolitana da capital de Minas Gerais. A área é composta por três localidades: Ravena, mais central (726 habitantes), Ravenópolis (1.057 habitantes) e Lava-pés (436 habitantes), perfazendo um total de 2.200 habitantes, o que significa um aumento de $52,8 \%$ da população em relação ao observado no ano de 1980 . A região é banhada pelos córregos Florêncio, Lava-pés e Rio Vermelho, que são ainda utilizados para algumas atividades de subsistência como retirada de areia, agricultura e pesca como lazer. Existem também algumas lagoas na região que são utilizadas para fins recreativos. A principal atividade exercida pela população de Ravena está ligada à plantação e ao comércio de banana, além de profissionais autônomos e trabalhadores ligados a uma fábrica de laticínios instalada às margens da BR-381, distante poucos quilômetros da entrada da localidade. Muitas pessoas trabalham em Belo Horizonte ou em cidades vizinhas, fato que torna Ravena um distrito com características de cidade dormitório. Na Figura 1, encontra-se a localização do distrito de Ravena com os pontos de coleta identificados.

Levantamento sócio-econômico e de contato com água

Antes do início do estudo foram realizadas palestras explicativas nas escolas e igrejas para a comunidade em geral. Foi também realizada uma parceria com os agentes do posto de saúde local (Programa Saúde da Família - PSF), na mobilização da população. Os dados cadastrais utilizados para a aplicação do inquérito entre a população foram obtidos no posto de saúde local. Cada pessoa ou responsável, no caso de crianças menores de nove anos, respondeu a um questionário de contato com água em que eram levantados a freqüência e o motivo do contato, além de informações sobre idade, gênero, escolaridade e ocupação. Em um segundo questionário, foram obtidos dados sobre moradia, renda familiar, abastecimento de água, destino dos dejetos e infra-estrutura da casa. Os questionários foram aplicados em papel entre os meses de março e novembro de 2007, com a ajuda de pessoas da própria comunidade, treinadas para o trabalho. Este estudo foi de base populacional e não amostral.

\section{Exame coproparasitológico}

Amostras de fezes da população do distrito foram coletadas para a realização do exame coproparasitológico no período de março a novembro de 2007. Foram examinadas duas lâminas de uma única amostra de fezes, pelo método de Kato-Katz 13. Foi considerado caso a pessoa que apresentou ao menos um ovo de S. mansoni em uma das lâminas. Os resultados dos exames foram repassados para o responsável pelo PSF que se encarregou do envio à população $\mathrm{e}$ do tratamento. Foi disponibilizado pelo Centro de Pesquisa René Rachou, Fundação Oswaldo Cruz (CPqRR/FIOCRUZ), o Praziquantel, medicamento recomendado pelo Ministério da Saúde para o tratamento da esquistossomose mansoni.

\section{Levantamento malacológico e exame de moluscos}

No período de maio a novembro de 2007, foram realizadas capturas de moluscos em todas as coleções hídricas descritas no estudo de 1980 11, 
Distrito de Ravena, Município de Sabará, Minas Gerais, Brasil, com os pontos de coleta de moluscos e de colocação de camundongos-sentinela.

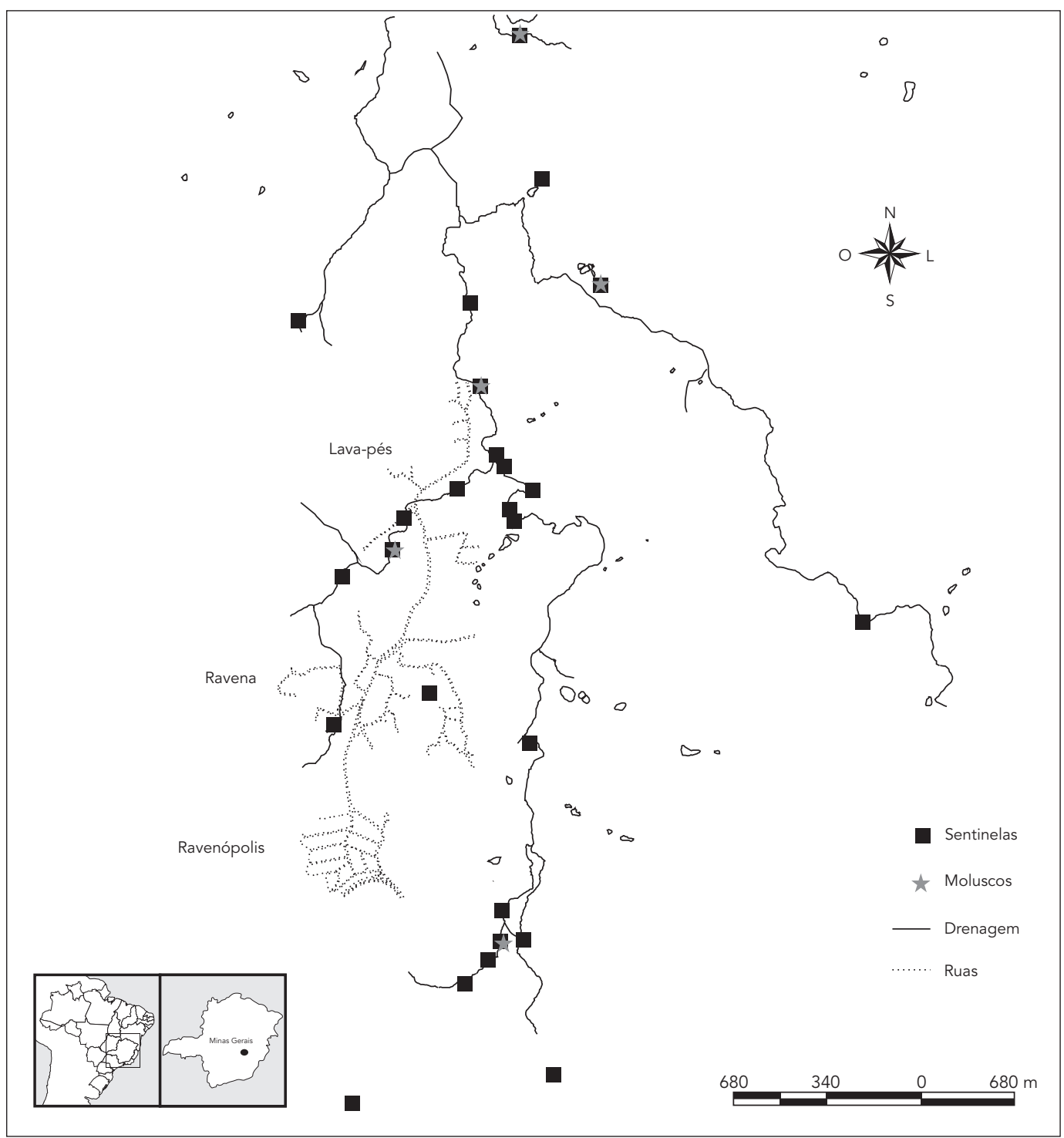

além das informadas pela população nos questionários de contato com água, somando um total de 12 locais de coleta. Para a coleta foi utilizada uma concha metálica perfurada que era mergulhada na água dez vezes a cada dez passos 14 . Os moluscos coletados foram devidamente armazenados em sacos plásticos, etiquetados e levados ao laboratório para exame e identificação específica.
Os moluscos foram submetidos a exames de detecção de cercárias e/ou esporocistos por estímulo luminoso e por esmagamento. De cada ponto onde foram coletados moluscos, foram separados cinco exemplares para identificação específica 15. 


\section{Sentinelas biológicos}

Camundongos sentinelas foram introduzidos mensalmente em cinco pontos das coleções hídricas, identificados pela população como locais de contato com água, no período de maio a outubro (Figura 1). Dez camundongos foram acondicionados, individualmente, em gaiolas de tela metálica com $3,5 \mathrm{~cm}$ de altura por $10 \mathrm{~cm}$ de diâmetro, munidas com tampas plásticas, fixadas em flutuadores de madeira e isopor com $50 \mathrm{~cm}$ de lado 16. Os camundongos foram introduzidos nas coleções hídricas, por uma hora, entre o período de 10:30h e 15h, por três dias consecutivos. Decorridos cinqüenta dias da exposição, foi feita a perfusão do sistema porta, para possível recuperação de vermes de S. mansoni 17 .

\section{Análise estatística}

A associação entre infecção por S. mansoni e variáveis relacionadas a características sócio-econômicas e contato com a água foi verificada por meio de estimativas de odds ratio (OR) obtidas por um ajuste de regressão logística múltipla não condicional, tendo como variável resposta a presença de esquistossomose e como variáveis preditoras as co-variáveis acima mencionadas. Inicialmente, foram ajustados modelos univariados para cada co-variável estudada. Aquelas cuja associação com a resposta apresentou valor de p menor que 0,2 foram selecionadas para o modelo múltiplo. Permaneceram no modelo final as co-variáveis cuja associação com a variável resposta apresentou valor de $\mathrm{p}$ menor que 0,05 .

As análises foram realizadas utilizando os programas Minitab versão 13.0 (Minitab Inc., State College, Estados Unidos) e SPSS versão 11.5 (SPSS Inc., Chicago, Estados Unidos).

Verificou-se a associação entre a ocorrência de esquistossomose na população estudada e a posição espacial de suas casas por meio do ajuste de um modelo aditivo generalizado 18 , tendo como variável resposta a presença da doença, e como variável preditora um spline cúbico bidimensional do vetor de coordenadas geográficas das moradias analisadas. Esta análise foi feita utilizando-se o programa R 2.7.0, library mgcv (The R Foundation for Statistical Computing, Viena, Áustria; http://www.r-project.org).

\section{Comitê de ética}

O Comitê de Ética em Pesquisa em Seres Humanos do CPqRR/FIOCRUZ, de acordo com as atribuições da Resolução $n^{\circ}$. 196/96 do Conselho Nacional de Saúde, homologou o projeto de pes- quisa proposto com o parecer $n^{\circ}$. 15/2007-CEPSH-CPqRR.

\section{Resultados}

\section{Caracterização da população}

Foram aplicados 2.019 (91\%) questionários individuais e 555 (96\%) questionários sócio-econômicos. Apenas os questionários das 1.591 pessoas $(72 \%)$ que enviaram amostras de fezes foram considerados para a análise.

Entre as residências pesquisadas nas três localidades, 97\% possuíam água tratada e 90\%, esgoto canalizado sem tratamento, sendo os dejetos lançados in natura nos córregos. A infra-estrutura das residências quanto ao destino dos dejetos e abastecimento de água tratada nas localidades de Ravena encontra-se na Tabela 1. Em relação à renda familiar, $67,2 \%$ da população declararam receber entre 1 e 3 salários mínimos e apenas $6,1 \%$ declarou renda superior a seis salários. Além disso, 89,1\% das moradias são próprias e $85,4 \%$ apresentavam instalação sanitária no interior das residências. As três localidades apresentaram características semelhantes, observadas na Tabela 1 .

\section{Prevalência da esquistossomose}

Dos 2.220 habitantes de Ravena, 1.591 (72\%) foram submetidos ao exame coproscópico: 482 (66\%) na localidade de Ravena, 785 (74\%) em Ravenópolis e 324 (74\%) em Lava-pés. Foram considerados os questionários individuais de contato com água apenas dos indivíduos examinados.

A prevalência da esquistossomose no distrito foi de 2,5\% (38 pessoas) com carga parasitária média de 21 ovos por grama de fezes. Durante a realização da pesquisa, não houve a notificação de nenhum caso grave da doença. Não houve diferença significativa na prevalência entre as três localidades. Nas análises univariadas, em relação à infecção para as três localidades, não houve associação da infecção por $S$. mansoni com o gênero, (17 mulheres para 21 homens).

As faixas etárias 15 a 19 e 20 a 29 anos apresentaram associação com a infecção por S. mansoni (Tabela 2). Pessoas com idade entre 15 e 19 anos apresentaram $\mathrm{OR}=2,3(\mathrm{p}=0,089)$ em relação às pessoas com mais de 30 anos de idade, e os indivíduos na faixa etária dos 20 aos 29 anos apresentaram $\mathrm{OR}=3,2(\mathrm{p}=0,002)$. Em Lava-pés, na análise univariada, as co-variáveis que apresentaram associações significativas com a infecção por S. mansoni foram: nadar $(\mathrm{p}=0,100)$; atravessar córrego $(p=0,044)$; trabalhar na lavoura 
Infra-estrutura das residências quanto ao destino dos dejetos e abastecimento de água tratada nas localidades de Ravena, Município de Sabará, Minas Gerais, Brasil, 2007.

\begin{tabular}{|c|c|c|c|}
\hline \multirow[t]{2}{*}{ Infra-estrutura das residências } & \multicolumn{3}{|c|}{ Localidades de Ravena } \\
\hline & Lava-pés (\%) & Ravena (\%) & Ravenópolis (\%) \\
\hline Água potável & 95,5 & 98,9 & 96,0 \\
\hline \multicolumn{4}{|l|}{ Destino dos dejetos } \\
\hline Rede de esgoto & 69,4 & 92,0 & 97,6 \\
\hline Fossa rudimentar & 11,1 & 7,0 & 0,8 \\
\hline
\end{tabular}

Prevalência da esquistossomose no distrito de Ravena, Município de Sabará, Minas Gerais, Brasil, segundo faixa etária da população em 2007.

\begin{tabular}{|c|c|c|c|c|c|c|c|c|}
\hline & \multicolumn{2}{|c|}{ Resultado de exame de fezes * } & \multirow[t]{2}{*}{ Total } & \multirow[t]{2}{*}{ Prevalência } & \multirow[t]{2}{*}{ Valor de $p$} & \multirow[t]{2}{*}{ OR } & \multicolumn{2}{|c|}{ IC95\% } \\
\hline & Negativo & Positivo & & & & & $\begin{array}{l}\text { Limite } \\
\text { inferior }\end{array}$ & $\begin{array}{l}\text { Limite } \\
\text { superior }\end{array}$ \\
\hline Faixa etária (anos) & & & & & 0,022 & & & \\
\hline $0-4$ & 120 & 0 & 120 & 0,0 & 0,996 & 0,0 & 0,0 & \\
\hline $5-9$ & 139 & 0 & 139 & 0,0 & 0,996 & 0,0 & 0,0 & \\
\hline $10-14$ & 149 & 1 & 150 & 0,7 & 0,296 & 0,3 & 0,0 & 2,6 \\
\hline $15-19$ & 131 & 6 & 137 & 4,4 & 0,089 & 2,3 & 0,9 & 6,1 \\
\hline $20-29$ & 253 & 16 & 269 & 5,9 & 0,002 & 3,2 & 1,6 & 6,5 \\
\hline$\geq 30$ & 757 & 15 & 772 & 1,9 & & 1,0 & & \\
\hline
\end{tabular}

* Método Kato-Katz.

$(\mathrm{p}=0,193)$; e retirar areia $(\mathrm{p}=0,081)$. O resultado das análises univariadas para a localidade de Ravena foi semelhante ao de Lava-pés. Em Ravenópolis, além das co-variáveis que apresentaram associação em Lava-pés e Ravena, foram incluídas as co-variáveis: tomar banho, lavar vasilha e ocupação de trabalhador rural. Nesta localidade, o indivíduo que exerce atividades de um trabalhador rural apresenta $\mathrm{OR}=7,8(\mathrm{p}=0,065)$.

\section{Análise múltipla}

Ajustou-se o modelo logístico múltiplo, que foi iniciado com as co-variáveis selecionadas na etapa univariada. As variáveis associadas ao risco de infecção por S. mansoni no distrito de Ravena foram: faixa etária $(\mathrm{p}=0,022)$, tipo de ocupação $(\mathrm{p}<0,001)$ e atravessar córrego $(\mathrm{p}<0,001)(\mathrm{Ta}-$ bela 3). Pessoas na faixa etária de 15 a 19 e 20 a 29 anos apresentam, respectivamente, $\mathrm{OR}=2,3$ (intervalo de 95\% de confiança - IC95\%: 0,9-6,1) e OR = 3,2 (IC95\%: 1,6-6,5) quando comparadas às pessoas com mais de 30 anos de idade. Pesso- as que atravessam córregos com uma frequência mensal ou maior têm OR = 4,6 (IC95\%: 2,1-10,2). Outro fator de risco associado à contaminação por S. mansoni em Ravena está ligado ao tipo de ocupação. Trabalhadores rurais ou braçais apresentam OR = 4,8 (IC95\%: 2,4-9,9) comparados a outros trabalhadores.

\section{Estimativa da espacialização do OR}

As estimativas de OR em função do espaço, obtidas na tentativa de ajustar-se o modelo aditivo generalizado acima definido, não apresentaram significância estatística. Em outras palavras, não parece haver associação significativa entre a existência de esquistossomose e os locais de moradia da população estudada. A Figura 2 apresenta a espacialização do OR obtida, subdividindo-se a área de estudo por meio de uma grade 100 x 100. Para cada retângulo dessa grade, a imagem informa, por intermédio de um gradiente de cores do branco (baixo OR) ao preto (alto OR), a estimativa da razão entre o odds presença/ausência 
Variáveis associadas ao risco de infecção por Schistosoma mansoni no distrito de Ravena, Município de Sabará, Minas Gerais, Brasil, 2007.

\begin{tabular}{|c|c|c|c|c|c|c|c|c|}
\hline \multirow[t]{2}{*}{ Co-variáveis } & \multicolumn{2}{|c|}{ Resultado de exame de fezes * } & \multirow[t]{2}{*}{ Total } & \multirow[t]{2}{*}{ Prevalência } & \multirow[t]{2}{*}{ Valor de $p$} & \multirow[t]{2}{*}{ OR } & \multicolumn{2}{|c|}{ IC95\% } \\
\hline & Negativo & Positivo & & & & & $\begin{array}{l}\text { Limite } \\
\text { inferior }\end{array}$ & $\begin{array}{l}\text { Limite } \\
\text { superior }\end{array}$ \\
\hline Faixa etária (anos) & & & & & 0,022 & & & \\
\hline $0-4$ & 120 & 0 & 120 & 0,0 & 0,996 & 0,0 & 0,0 & \\
\hline $5-9$ & 139 & 0 & 139 & 0,0 & 0,996 & 0,0 & 0,0 & \\
\hline $10-14$ & 149 & 1 & 150 & 0,7 & 0,296 & 0,3 & 0,0 & 2,6 \\
\hline $15-19$ & 131 & 6 & 137 & 4,4 & 0,089 & 2,3 & 0,9 & 6,1 \\
\hline $20-29$ & 253 & 16 & 269 & 5,9 & 0,002 & 3,2 & 1,6 & 6,5 \\
\hline$\geq 30$ & 757 & 15 & 772 & 1,9 & & 1,0 & & \\
\hline Ocupação & & & & & 0,000 & & & \\
\hline Outros & 1.413 & 25 & 1.438 & 1,7 & & 1,0 & & \\
\hline Trabalhador rural/braçal & 140 & 12 & 152 & 7,9 & & 4,8 & 2,4 & 9,9 \\
\hline Atravessar córrego & & & & & 0,000 & & & \\
\hline Nunca & 1.378 & 25 & 1.403 & 1,8 & & 1,0 & & \\
\hline Anual/Semestral & 67 & 4 & 71 & 5,6 & 0,031 & 3,3 & 1,1 & 9,7 \\
\hline Mensal ou mais & 107 & 9 & 116 & 7,8 & 0,000 & 4,6 & 2,1 & 10,2 \\
\hline
\end{tabular}

* Método Kato-Katz.

\section{Figura 2}

Espacialização da estimativa do odds ratio (OR) em relação à presença e à ausência de esquistossomose em Ravena, Município de Sabará, Minas Gerais, Brasil, 2007.

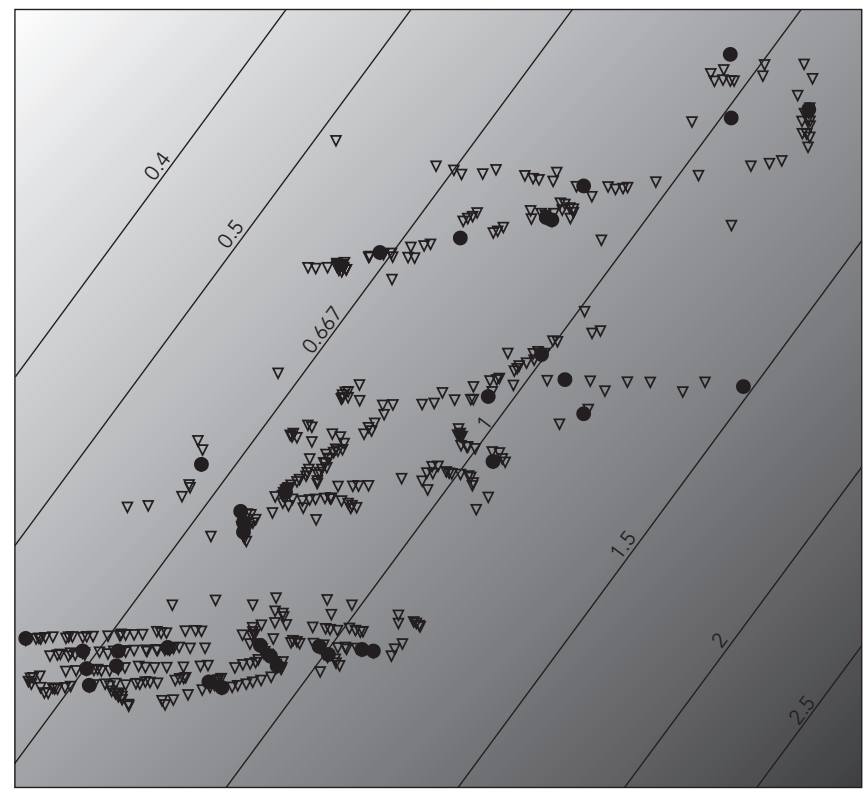

- Casos

$\nabla$ Controles de esquistossomose no retângulo e o odds presença/ausência de esquistossomose na área de estudo como um todo. A pequena variabilidade da figura reforça a idéia de independência entre presença de esquistossomose e local de moradia dentro da área estudada.

\section{Levantamento malacológico}

Foram capturados 5.630 exemplares de Biomphalaria sp. em 12 pontos de coleta nos córregos Florêncio, Rio Vermelho e Lava-pés, do balneário do Zé Rocha, Lagoa do Nô, Siqueira, Lagoa do Zinho, Bica do Chafariz, Maquiné, Acampamento Batista, Vala da Bomba e balneário do km 30 . Do total de moluscos capturados, 2.788 foram identificados como B. tenagophila, 2.086 como B. glabrata e 756 como B. straminea. Entre os moluscos capturados, seis exemplares de B. glabrata, originários do córrego Lava-pés, encontravam-se infectados com S. mansoni. B. straminea foi encontrada apenas em uma coleção hídrica "Zé Rocha”, utilizada para lazer nos finais de semana. Nos córregos Florêncio e Rio Vermelho, foi encontrada a B. tenagophila. Em um trecho do córrego Lava-pés e em mais cinco coleções hídricas pesquisadas (Siqueira, Lagoa do Zinho, Bica do Chafariz, Vala da Bomba e balneário km 30), foram coletados B. glabrata. Neste estudo foi observado que locais anteriormente ocupados pela B. glabrata, como Rio Vermelho, hoje são dominados por B. tenagophila. 


\section{Sentinelas biológicos}

As sentinelas biológicas foram introduzidas em cinco pontos de diferentes coleções hídricas muito citadas pela população no questionário de contato com água. São eles: Porto de Areia (Córrego Florêncio), Campinho (córrego Lava-pés), Lavadeiras (Córrego Rio Vermelho), balneário do Zé Rocha e Siqueira. Durante o período da pesquisa, foram utilizados 300 camundongos, entre os meses de maio e outubro de 2007. Não foi observada a infecção por $S$. mansoni em nenhum dos animais.

\section{Discussão}

Há 27 anos, quando pesquisadores realizaram um estudo sobre esquistossomose em Ravena, foi detectada uma prevalência de $36,7 \%$. Nas localidades de Lava-pés a prevalência foi de 63,9\%, em Ravena 42,6\% e em Ravenópolis 20,1\%. A primeira apresentava as piores condições sanitárias, sem abastecimento de água, e morar nessa área era um fator de risco para a endemia 11 . Hoje, Lava-pés possui $95,5 \%$ das casas abastecidas com água potável e 80,5\% delas destinam seus dejetos para a rede de esgoto ou fossa rudimentar. Atualmente, não há diferença entre as três localidades em relação ao risco de contrair esquistossomose. Os padrões de contato com água em Ravena modificaram-se ao longo dos anos. A maioria da população não tem mais o hábito de utilizar água das coleções hídricas para as tarefas domésticas (lavar roupa e/ou vasilha), provavelmente devido aos investimentos com o fornecimento de água tratada. A poluição dos corpos d'água é evidente, pois o esgoto das casas é coletado e lançado in natura sem nenhum tratamento. Outros estudos indicam que melhorias na infra-estrutura associadas à educação e ao tratamento específico mostram-se como as medidas mais eficazes e duradouras para reduzir a prevalência e a morbidade da esquistossomose 19 .

No estudo de 1980 em Ravena ${ }^{11}$, observou-se associação da infecção por $S$. mansoni com o gênero masculino, entretanto, no presente, estudo essa associação não foi observada. Em relação à faixa etária, dos 38 casos de esquistossomose detectados no distrito, 37 ocorreram em pessoas maiores de 15 anos e um caso em uma criança de 12 anos. Essa criança reside em Lava-pés e relatou ter o hábito de nadar e atravessar o córrego de mesmo nome, onde foram capturados exemplares de B. glabrata infectados. Apesar desse caso, o resultado pode indicar uma diminuição significativa da transmissão da esquistossomose na área urbana de Ravena.
Os demais 37 participantes positivos podem ser considerados como casos não curados de tratamentos anteriores, reinfectados, com baixa carga parasitária ou infectados em outras áreas. Assim, a metodologia recomendada pelo Programa de Controle da Esquistossomose, utilizando escolares de 7 a 14 anos para definir prevalência, deve ser aplicada com cautela nas áreas de prevalência abaixo de $5 \%$, sobretudo se a metodologia utilizada for a mesma dos programas de controle da Secretaria de Estado de Saúde de Minas Gerais, que é de apenas uma lâmina por amostra de fezes pelo método de Kato-Katz. Há estudos que confirmam que a prevalência é subestimada pela baixa sensibilidade do método de diagnóstico 20. Para minimizar o problema, os autores sugerem o aumento do número de amostras e de lâminas para o diagnóstico. Dessa forma, a prevalência poderá se aproximar do valor real para melhorar a eficiência do controle da esquistossomose em áreas de baixa endemicidade. Neste trabalho, foi seguida a mesma metodologia adotada em 1980: duas lâminas para uma amostra de fezes, mas provavelmente nas duas ocasiões ocorreu uma subestimativa da doença. No estudo de 1980, a prevalência da esquistossomose em Ravena era de $36,7 \%$ e as crianças na faixa etária de 0 a 14 anos apresentaram uma prevalência de $11,62 \% 11$. Hoje, a prevalência no distrito é de $2,5 \%$ e de $0,7 \%$ na faixa etária de 0 e 14 anos, com apenas uma criança eliminando ovos de $S$. mansoni. Foi observado que as pessoas não mantêm mais o hábito de utilizar as águas das coleções hídricas para tarefas domésticas como há 27 anos. O principal motivo para a mudança de hábito foi a introdução de água potável em mais de 95\% das residências do distrito. Contudo, em Lavapés, 30,6\% das residências continuam a lançar in natura o esgoto no córrego de mesmo nome o que pode explicar o encontro da criança de 12 anos infectada. Por outro lado, deve ser lembrada a inexistência de tratamento do esgoto no distrito, cujas redes de esgoto são direcionadas para o Rio Vermelho. Em outros estudos, como o realizado em Taquaraçu de Minas, Estado de Minas Gerais 19, também foi observada uma queda significativa na prevalência da esquistossomose após a implantação de medidas de controle como o tratamento da população e o fornecimento de água potável intradomiciliar.

No levantamento malacológico realizado de maio a outubro de 1980, foram coletados 8.989 exemplares de Biomphalaria, sendo 3.812 exemplares de B. glabrata em 12 coleções hídricas e 5.177 de B. tenagophila em um único curso de água. No presente estudo, as coletas foram realizadas no período de maio a novembro de 
2007 em três córregos (Lava-pés, Rio Vermelho e Florêncio) e em mais nove coleções hídricas. Foram coletados 5.630 exemplares de moluscos. Neste levantamento, foi registrada pela primeira vez a ocorrência de $B$. straminea no distrito de Ravena, no balneário do Zé Rocha. A espécie $B$. glabrata foi encontrada no Córrego Lava-pés e em mais cinco coleções hídricas (Siqueira, Lagoa do Zinho, Bica do Chafariz, Vala da Bomba e Km 30) e a espécie B. tenagophila em três locais (Rio Vermelho, do Florêncio e Maquiné).

Foi possível observar que a B. tenagophila, que ocupava apenas uma coleção hídrica (vala que não existe mais), atualmente foi encontrada em três locais. Essa dispersão pode ter ocorrido no período das chuvas, quando há inundações que podem causar o deslocamento de planorbídeos 21 . A dispersão de moluscos também pode ser verificada passivamente, por meio da inserção de peixes e plantas aquáticas 22,23,24. Além dessas possibilidades há a introdução de espécies pela construção civil, como o caso de Ilha Bela no litoral de São Paulo, Brasil 25 onde a terra empregada na construção de um aterro foi retirada de uma localidade de São Sebastião, dominada por B. tenagophila, onde existiam tanques de piscicultura que provavelmente abrigavam $B$. straminea 26.

B. tenagophila foi encontrada em coleções d'água anteriormente habitadas por B. glabrata (Rio Vermelho). Em outras áreas, observações semelhantes já foram relatadas. Uma população de B. tenagophila proveniente de São Paulo e introduzida em uma lagoa do bairro Baleia em Belo Horizonte, Minas Gerais, substituiu, cerca de cinco anos, uma população autóctone de $B$. glabrata 21 . Foi observado também que uma população de B. straminea deslocou uma população de B. glabrata nos arredores de Recife, Pernambuco, Brasil 27.

Suspeita-se que, em Ravena, a B. glabrata seja a única responsável pela manutenção da doença. Porém, a presença de B. tenagophila e B. straminea reforça a necessidade de vigilância constante no distrito uma vez que esses moluscos são responsáveis pela transmissão do $S$. mansoni em várias outras regiões do país, como em São Paulo e Nordeste brasileiro, respectivamente.
Apesar de nenhum camundongo-sentinela ter sido infectado por $S$. mansoni, foi encontrado ao longo do córrego Lava-pés $B$. glabrata liberando cercárias do parasita. Como não existe tratamento de esgoto, as fezes dos infectados alcançam as coleções hídricas, fechando assim o ciclo. A dificuldade de infectar camundongossentinela e de encontrar caramujos infectados pode estar relacionada à baixa carga parasitária da população que foi em média de 21 ovos por grama de fezes.

Ravena apresentou uma resposta positiva às intervenções de controle da esquistossomose, mas é uma área de risco em potencial, pois apresenta fatores ambientais e ecológicos favoráveis à manutenção da doença. Para que os níveis de prevalência continuem baixos, será necessário investir em saneamento, educação, além de tratamento dos infectados. Apesar do alto índice de casas com água potável, a infra-estrutura em alguns locais do distrito ainda é bastante precária. A falta de opção de lazer e/ou a falta de informação, faz com que particularmente os jovens adultos, entre 20 e 45 anos, tenham contato com as coleções hídricas da região, correndo o risco de se infectarem. Possivelmente isso ocorre porque as crianças permanecem grande parte do tempo em casa ou nas proximidades desta com a presença dos pais. O fator de maior risco detectado neste estudo foi o tipo de ocupação das pessoas, especialmente trabalhadores rurais que ficam mais expostos ao risco de infecção por terem contato diário com água, tanto para o tratamento dos animais quanto para o cultivo de pequenas plantações. Outro fator relacionado ao risco de infecção é o hábito de as pessoas atravessarem córregos, apesar de acharem que a única maneira de se infectar com $S$. mansoni é permanecer por longo tempo dentro de rios ou lagoas e o simples fato de molhar os pés não apresenta nenhum risco. Portanto, campanhas informativas nas escolas, igrejas e centros comunitários, com a ajuda dos agentes do PSF, podem ser eficientes para mudar essa prática, contribuindo, assim, para a redução do número de indivíduos infectados. 


\section{Resumo}

Os programas de controle da esquistossomose no Brasil têm contribuído para a redução da prevalência e das formas graves da doença, mas não têm impedido que novos focos apareçam, especialmente nas áreas periféricas dos grandes centros urbanos do país. O presente artigo tem como objetivo avaliar a prevalência da esquistossomose e a presença de hospedeiros intermediários do Schistosoma mansoni no distrito de Ravena, Município de Sabará, Minas Gerais, Brasil, após 27 anos da implantação do sistema de água tratada e do tratamento específico da população infectada. Ra vena apresentou uma resposta positiva às intervenções de controle da esquistossomose, apresentando uma prevalência de 2,5\%. Porém, é uma área de risco em potencial, pois apresenta fatores ambientais e ecológicos favoráveis à manutenção da doença. Para que os níveis de prevalência continuem baixos, será necessário investir em saneamento, educação em saúde além de tratamento dos infectados.

Controle de Doenças Transmissíveis; Esquistossomose mansoni; Moluscos

\section{Colaboradores}

C. H. Vasconcelos, P. C. M. Cardoso, C. L. Massara e O. S. Carvalho participaram da concepção do trabalho, das coletas e análise dos dados e redação do texto. W. C. Quirino e G. L. Amaral contribuíram nas coletas dos dados e na revisão do manuscrito. R. Cordeiro colaborou na revisão do manuscrito e nas análises estatística e espacial realizadas.

\section{Agradecimentos}

Ao José Geraldo Amorim do Laboratório de Helmintologia e Malacologia Médica, Centro de Pesquisa René Rachou, Fundação Oswaldo Cruz (CPqRR/FIOCRUZ), por realizar a captura dos moluscos e o diagnóstico dos exames de fezes. Ao pesquisador Martin Johannes Enk pelas dicas que contribuíram para a apresentação deste trabalho e à Fundação de Amparo à Pesquisa do Estado de Minas Gerais (FAPEMIG processo ${ }^{\circ}$. CRA APQ4178-5.02/07) pelo financiamento do projeto.

\section{Referências}

1. Katz N. Schistosomiasis control in Brazil. Mem Inst Oswaldo Cruz 1998; 93 Suppl 1:33-5.

2. Rabello A, Enk M. Progress towards the detection of schistosomiasis. Geneva: Special Programme for Research and Training in Tropical Diseases, World Health Organization; 2005.

3. Carvalho OS, Massara CL, Silveira Neto HV, Guerra HL, Caldeira RL, Mendonca CLF, et al. Re-evaluation of schistosomiasis mansoni in Minas Gerais, Brazil - II. Alto Paranaiba mesoregion. Mem Inst Oswaldo Cruz 1997; 92:141-2.

4. Graeff-Teixeira C, Anjos CB, Oliveira VC, Velloso CEP, Fonseca MBS, Valar C, et al. Identification of a transmission focus of Schistosoma mansoni in the southernmost Brazilian state, Rio Grande do Sul. Mem Inst Oswaldo Cruz 1999; 94:9-10.

5. Enk MJ, Caldeira RL, Carvalho OS, Schall VT. Rural tourism as risk factor for the transmission of schitosomiasis in Minas Gerais, Brazil. Mem Inst Oswaldo Cruz 2004; 99 Suppl 1:105-8.

6. Carmo EH, Barreto ML. Esquistossomose mansônica no estado da Bahia, Brasil: tendências históricas e medidas de controle. Cad Saúde Pública 1994; 10:425-39.
7. Kloetzel K, Chieffi PP, Carrilho FJ. Environmental intervention as a tool for control of schistosomiasis: suggestion from a field study in Northeast Brazil. Cad Saúde Pública 1994; 10 Suppl 2: 337-44

8. Amorim MN, Rabello A, Contreras RL, Katz N. Epidemiological characteristics of Schistosoma mansoni infection in rural and urban endemic areas of Minas Gerais, Brazil. Mem Inst Oswaldo Cruz 1997; 92:577-80.

9. Moza PG, Pieri OS, Barbosa CS, Rey L. Fatores sócio-demográficos e comportamentais relacionados à esquistossomose em uma agrovila da zona canavieira de Pernambuco, Brasil. Cad Saúde Pública 1998; 14:107-15.

10. Gazzineli A, Velasquez-Melendez G, Crawford SB, LoVerde PT, Correa-Oliveira R, Kloos H. Socioeconomic determinants of schistosomiasis in a poor rural area in Brazil. Acta Trop 2006; 99:260-71.

11. Coura-Filho P, Farah MWC, Resende DF, Lamartine SS, Carvalho OS, Katz N. Determinantes ambientais e sociais da esquistossomose mansoni em Ravena, Minas Gerais, Brasil. Cad Saúde Pública 1995; 11:254-65. 
12. Coura-Filho P, Rocha RS, Lamartine SS, Farah MWC, Resende DF, Costa JO, et al. Control of schistosomiasis mansoni in Ravena (Sabará, state of Minas Gerais, Brasil) through water supply and quadrenial treatments. Mem Inst Oswaldo Cruz 1996; 91:659-64.

13. Katz N, Chaves A, Pellegrino J. A simple device for quantitative stool thick-smear technique in schistosomiasis mansoni. Rev Inst Med Trop São Paulo 1972; 14:397-400.

14. Oliver L, Schneiderman M. A method for estimating the density of aquatic snail populations. Exp Parasitol 1956; 5:109-17.

15. Paraense WL. Estado atual da sistemática dos planorbídeos brasileiros. Arquivos do Museu Nacional 1975; 55:105-28.

16. Paulini E, Dias EP. Resultados de três anos de controle da esquistossomose em Belo Horizonte (MG). Rev Bras Malariol Doenças Trop 1971; 24:151-72.

17. Pellegrino J, Katz N. Experimental chemothrapy of schistosomiasis mansoni. Adv Parasitol 1968; 6:233-90.

18. Bailey TC, Cordeiro R, Lourenço RW. Semiparametric modeling of the sapatial distribution of occupational accident risk in the casual labor market, Piracicaba, southeast Brazil. Risk Anal 2007; 27:1-12.

19. Coura-Filho P. Participação popular no controle da esquistossomose através do Sistema Único de Saúde (SUS), em Taquaraçu de Minas, (Minas Gerais, Brasil), entre 1985-1995: construção de um modelo alternativo. Cad Saúde Pública 1998; 14 Suppl 2:111-22.
20. Enk MJ, Lima ACL, Massara CL, Coelho PMZ, Schall VT. A combined strategy to improve the control of Schistosoma mansoni in areas of low prevalence in Brazil. Am J Trop Med Hyg 2008; 78:140-6.

21. Paraense WL. Planorbídeos hospedeiros intermediários do Schistosoma mansoni. In: Cunha AS, organizador. Esquistossomose mansoni. São Paulo: EdUSP; 1970. p. 13-30.

22. Corrêa LL, Corrêa MOA, Vaz JF, Silva RM, Yamanaka MT. Importância das plantas ornamentais dos aquários como veículos de propagação dos vetores do Schistosoma mansoni. Rev Inst Adolfo Lutz 1980; 40:89-96.

23. Madsen H, Frandsen F. The spread of freshwater snails including those of medical and veterinary importance. Acta Trop 1989; 46:139-46.

24. Guimarães CT, Souza CP, Soares DM, Araújo N, Schuster LMR. Occurrence of molluscs in aquaria of ornamental fishes in Belo Horizonte, Minas Gerais, Brazil. Mem Inst Oswaldo Cruz 1990; 85:127-9.

25. Teles HMS, Hiramoto RM, Oliveira JCN, Aveiro JA. Ocorrência de vetores da esquistossomose mansônica no litoral norte do Estado de São Paulo, Brasil. Cad Saúde Pública 2003; 19:1887-91.

26. Teles HMS. Distribuição de Biomphalaria straminea ao sul da região Neotropical, Brasil. Rev Saúde Pública 1996; 30:341-9.

27. Barbosa FS. Possible competitive displacement and evidence of hybridization between two Brazilian species of planorbid snails. Malacologia 1973; 14:401-8.

Recebido em 07/Mai/2008

Versão final reapresentada em 10/Out/2008

Aprovado em 17/Dez/2008 